\title{
Synthesis of Some Amino Acid and Peptide Conjugates and their Evaluation as Potential Anti-allergic and Anti-inflammatory Agents
}

\author{
A.M. Shalaby, O.I. Abd Al-Salam*, A. Kalmouch, S.E. \\ EL-Shihaby and M. Abdullah *** \\ Department of Peptide Chemistry, *Department of Applied \\ Organic Chemistry, National Research Center, 12622, Giza \\ and **Research Unit, Hi-care Pharmaceutical Co., Cairo, \\ Egypt.
}

\begin{abstract}
A NEW series of $N$-3-(2-furanyl) acryloyl, N-3-(5-methyl)-2(furanyl)-acryoyl amino acids, peptide and piprazines amids, were structurally designed and synthesized. About 23 compounds (5aw) were synthesized and their anti-allergic and anti-inflamatory activities were evaluated relative to those of Loratadine $\left(\right.$ Clorinix ${ }^{\circledR}$ ) and Diclofenac ${ }^{\circledR}$. All the obtained products showed good anti-allergic activities with the greatest activities recorded for compounds $(5 \mathrm{~g}, 5 \mathrm{~h}$, $5 \mathrm{j}, 5 \mathrm{k}, 5 \mathrm{~m}, 5 \mathrm{n}, 5 \mathrm{v}$ and $5 \mathrm{w})$. All compounds showed $\mathrm{LD}_{50}$ with safety margin. On the other hand most compounds have no anti-inflamatory activities except products (5d, 5e and 5p).
\end{abstract}

Keywords: 3-(2-Furanyl)acrylic acid, Amino acids, Piprazines and Anti-allerg and Anti-inflammatory.

The promising success of Youshinori Nishikawa et al. ${ }^{(1-3)}$ in designing new antiallergic drugs characterized as an antagonist of histamine as well as inhibitor for the generation of leukotrienes and prostaglandins, has stimulated a research interest. They synthesized orally active anti-allergic compounds having structure [A] with acrylamide segments responsible for inhibition of enzyme 5lipoxygenase which catalyzed the generation of chemical mediators like (leukotrienes) from arachidonic acid and cause allergy. Tranilast ${ }^{\circledR}$, a drug contains $\beta$-phenylacrylamide moiety. The acrylamide moiety may contribute to mediator release inhibitory activity. $\beta$-Aryl and $\beta$-heteroarylacrylamides may also show promising activity. Consequently, they designed, the acrylamide derivatives, substituted with 4-piprazinyl moieties, which, on the other hand, are expected to possess anti-histaminic activity similar to drug Oxatomide ${ }^{\circledR}$. So the presence of piprazine moiety is responsible for this activity.

Their success led to synthesize new drug namely "Tagorizine" [N-[4-(4diphenylmethyl-1-piprazinyl) butyl]-3-(3-pyridyl)acrylamide] ${ }^{(4-7)}$.

In harmony with these findings, herein, some acryloyl/ amino acids and/or peptide and piperazine amides having structure [B] have been synthesized (Fig. 1). 
The addition of amino acids, peptide instead of alkyl chain was to introduce active moiety beside acrylamide and piprazine moieties. This is due to that amino acids and peptide play an outstanding role as growth factors, hormones, ionophores, immune peptides and toxins ${ }^{(8,9)}$.<smiles>[R4]C=CC(=O)NN=CN1CCN([R])CC1</smiles>

[A]<smiles>[R3]NC(=O)/C=C/c1ccc([R3])o1</smiles>

[B]

$$
\begin{aligned}
& \mathrm{n}=1-4 \\
& \mathrm{R}_{1}=\text { aryl or heteraryl } \\
& \mathrm{R}_{2}=\text { alkyl or aryl }
\end{aligned}
$$

$$
\begin{aligned}
& \mathrm{R}=\text { amino acid resiue } \\
& \mathrm{R}_{2}=\text { alkyl, ester or aryl } \\
& \mathrm{R}_{3}=\mathrm{H} \text { or } \mathrm{CH}_{3}
\end{aligned}
$$

Fig. 1. Structure design of the target products $[B]$.

Amino acids and peptides, being natural and multifunctional, their conjugates with biologically active agents which are generally synthetic organics are supposed to be more potent and particularly less toxic than their parent compounds ${ }^{(10-13)}$, also give better solubility, more favorable transport properties, pharmacologically adequate pattern and enzymatic degradation and general physiological compatibility.

Our previous work ${ }^{(14)}$ reported that some of the $\beta$-aryl and $\beta$ heteroarylacrylamides conjugated with amino acid, peptide and piprazinyl group displayed a markedly high anti-allergic activity.

The present study focused on enhancing this activity, and new series of amino acids and peptide conjugated with N-3-(2-furyl)acryloyl, N-3-(5-methyl-2furyl)acryloyl and piprazine derivatives were synthesized (Scheme 1). Also, the anti-allergic and anti-inflammatory activities were evaluated and promising results were obtained.

\section{Results and Discussion}

In the present study, $N$-3-(2-furanyl)acrylic acid was coupled with hydrochloride esters of glycine, L-valine, L-phenylalanine, L-tyrosinine and the dipeptide glycylglycine to afford $N$-3-(2-furanyl)acryloyl amino acid 3a-d and dipeptide 3e esters. Meanwhile, 3-(5-methyl)-2-furan acrylic acid was reacted with hydrochloride ester of glycine, L-valine and the dipeptide glycylglycine, to give the corresponding esters $3 \mathrm{f}-\mathrm{h}$, respectively (Scheme 1).

Coupling was performed via three methods: [i] the acid chloride method in which direct acylation is used; [ii] in situ active ester method (modified classical method) using 1-hydroxybenzotriazole (HOBt) and [iii] mixed anhydride method using ethyl chloroformate in the presence of $\mathrm{N}$-methylmorpholine as a catalyst.

Egypt. J. Chem. 56, No.5,6 (2013) 
The corresponding acid derivatives $4 \mathrm{a}-\mathrm{h}$, have been easily obtained in $80-90 \%$ yield by the alkaline hydrolysis of their corresponding esters $3 \mathrm{a}-\mathrm{h}$ under mild conditions and thin layer chromatography (TLC) control. Finally, each of the obtained acids 4a-h was coupled with 4-methylpiprazine, 4-ethoxycarbonylpiprazine and/or 1-(4-fluorophenyl)-piperazine to afford the corresponding designed products $N$-[(4-substituted-1- piperazinyl)-oxo- (alkyl and ethylcarbamoyl)] -3furanacrylamides 5a-w, respectively (Scheme 1).

The purity of the synthesized compounds as well as the starting materials was checked by thin layer chromatography (TLC) in an appropriate solvent system.

The structures of the synthesized compounds were confirmed by the inspection of their spectroscopic data, namely IR, ${ }^{1} \mathrm{H}-\mathrm{NMR}$ and EI-mass spectra, as well as, their elemental analyses (Tables 1 and 2). The infrared spectra indicated the expected absorption bands of the essential functional groups, in addition to their unique fingerprints. Thus, the aromaticity, amide, C-terminal carboxylic or ester groups were traced and located.

The aromaticity of the compounds was evident from their multiple absorptions $\sim 3100$ and $1600 \mathrm{~cm}^{-1}$; the medium stretching vibration involving carbon-to-carbon skeletal aromatic ring stretching appeared at $1580-1629 \mathrm{~cm}^{-1}$ and finally the weak aromatic C-H stretching at $2964-3084 \mathrm{~cm}^{-1}$. The secondary amide nature of the compounds was represented by amide band I absorption at $1647-1673 \mathrm{~cm}^{-1}$, reflecting a resonating $(\mathrm{N}-\mathrm{C}=\mathrm{O})$ stretching vibration.

The amide band II absorption, originating from a coupled $\mathrm{NH}$ bending and $\mathrm{C}$ $\mathrm{N}$ stretching vibration appeared at $1521-1578 \mathrm{~cm}^{-1}$.

The ${ }^{1} \mathrm{H}-\mathrm{NMR}$ spectra of the synthesized conjugates confirmed their hydrogen skeleton. The two vinyl protons $\left(\mathrm{H}_{\mathrm{a}}\right.$ and $\left.\mathrm{H}_{\mathrm{b}}\right)$ split one another into two doublets centered at $\delta \approx 6.5$ and $7.62 \mathrm{ppm}$, respectively, where downfield was assigned to the aromatic resonance for $\mathrm{H}_{\mathrm{a}}$. The proton $\mathrm{H}_{\mathrm{b}}$, being attached to the carbon, bearing the heteroaromatic ring, assigned the higher chemical shift due to the deshielding effect of an isotropic field degenerated by the electrons of the aromatic ring.

A coupling constant $J_{H H} \approx 16 \mathrm{~Hz}$, is a common value for trans proton-proton coupling across a double bond (E-configuration). Matching these absorptions from the spectra of the synthesized conjugates rendered facile the assignment of the remaining protons. Other signals at $\delta=7.5-7.62 \mathrm{ppm}$ for $\mathrm{H}_{\mathrm{b}}$, which was up-field due to the aromatic resonance for 3-(2-furanyl)acryloyl and 3-(5-methyl)-2-furanylacrylol residues, respectively. Methyl proton was assigned at $\delta=1.12 \mathrm{ppm}$ as a singlet.

The electron impact mass spectral data of the compounds were consistent with the proposed structures. Molecular ion peaks corresponding to the exact masses of the required formula were, generally, well represented with considerable abundances, indicating the relative stability of the compounds under electron bombardment. 

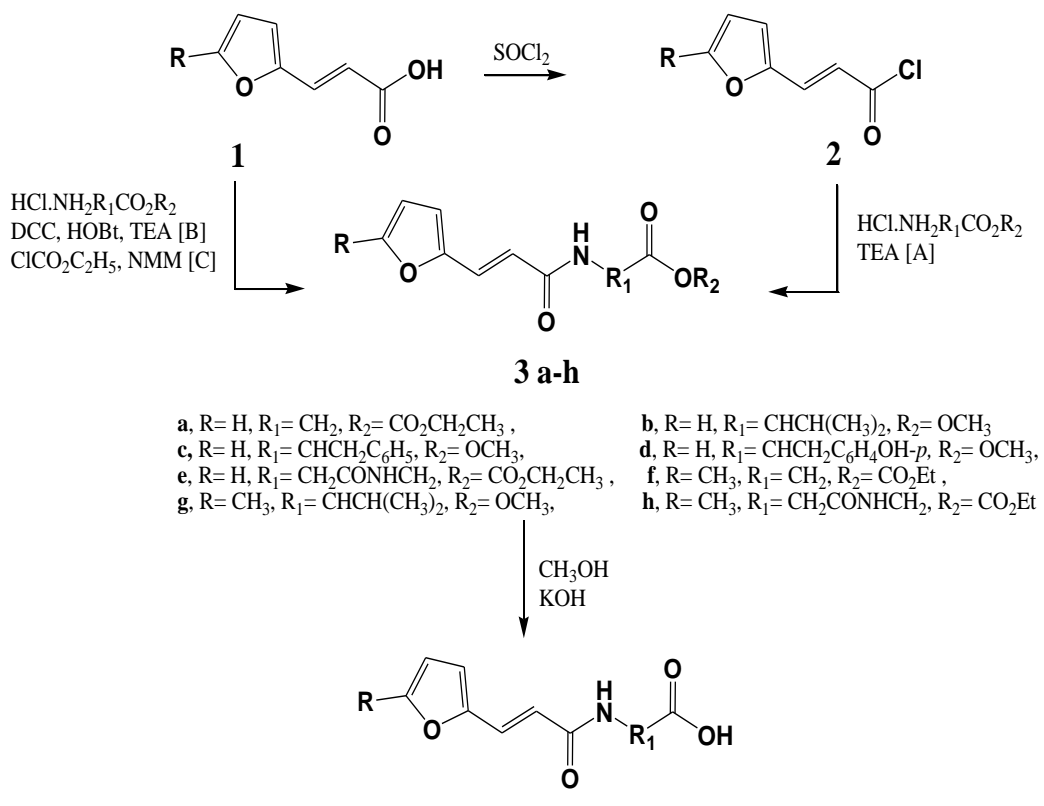

4 a-h

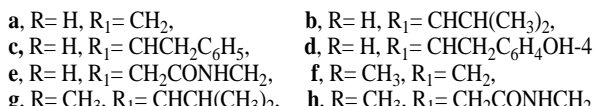

g, $\mathrm{R}=\mathrm{CH}_{3}, \mathrm{R}_{1}=\mathrm{CHCH}\left(\mathrm{CH}_{3}\right), \quad$ h, $\mathrm{R}=\mathrm{CH}_{3}, \mathrm{R}_{1}=\mathrm{CH}_{2} \mathrm{CONHCH}_{2}$

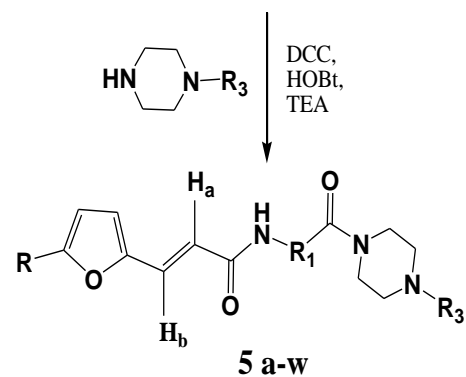

\begin{tabular}{|c|c|}
\hline 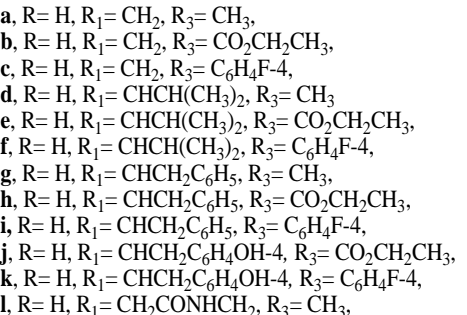 & 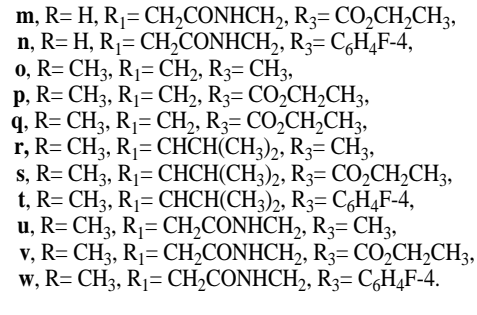 \\
\hline
\end{tabular}

Scheme 1

Egypt. J. Chem. 56, No.5,6 (2013) 
TABLE 1. Physical data of the prepared compounds 5a-w.

\begin{tabular}{|c|c|c|c|c|c|c|c|c|}
\hline \multirow{2}{*}{$\begin{array}{l}\text { Compd. } \\
\text { No. }\end{array}$} & \multirow{2}{*}{$\begin{array}{l}\text { Yield } \\
(\%)\end{array}$} & \multirow{2}{*}{$\underset{{ }^{0} \mathrm{C}}{\mathrm{Mp}}$} & \multirow{2}{*}{$\begin{array}{l}\text { Mol formula } \\
\text { (M. Wt.) }\end{array}$} & \multicolumn{3}{|c|}{$\begin{array}{l}\text { Elemental Analysis } \\
\text { (Calcd/Found) }\end{array}$} & \multirow{2}{*}{$\begin{array}{c}\mathbf{R}_{f} \\
\mathbf{x} 100 \\
\text { (Eluent) }\end{array}$} & \multirow{2}{*}{$\begin{array}{c}{[\alpha]_{\mathrm{D}}{ }^{25}} \\
(\mathbf{c}=0.5 \\
\text { methanol })\end{array}$} \\
\hline & & & & $\mathrm{C}$ & $\mathbf{H}$ & $\mathbf{N}$ & & \\
\hline \multirow[t]{2}{*}{$5 a$} & $39[\mathrm{~A}]$ & $168-$ & $\mathrm{C}_{14} \mathrm{H}_{19} \mathrm{~N}_{3} \mathrm{O}_{3}$ & 60.64 & 6.91 & 15.15 & 20 & \multirow{2}{*}{ - } \\
\hline & & 170 & 277.32 & 60.67 & 6.83 & 15.25 & $S_{1}$ & \\
\hline \multirow{2}{*}{$5 b$} & $35[\mathrm{~A}]$ & $170-$ & $\mathrm{C}_{16} \mathrm{H}_{21} \mathrm{~N}_{3} \mathrm{O}_{5}$ & 57.29 & 6.31 & 12.53 & 59 & \multirow{2}{*}{-} \\
\hline & $49[\mathrm{~B}]$ & 172 & 335.36 & 57.39 & 6.35 & 12.63 & $\mathrm{~S}_{2}$ & \\
\hline \multirow{2}{*}{$5 c$} & $35[\mathrm{~B}]$ & $180-$ & $\mathrm{C}_{19} \mathrm{H}_{20} \mathrm{FN}_{3} \mathrm{O}_{3}$ & 63.84 & 5.64 & 11.75 & 80 & \multirow{2}{*}{-} \\
\hline & $52[\mathrm{C}]$ & 200 & 357.15 & 63.22 & 5.60 & 11.85 & $S_{1}$ & \\
\hline \multirow{2}{*}{$5 \mathrm{~d}$} & $53[\mathrm{~B}]$ & $135-$ & $\mathrm{C}_{17} \mathrm{H}_{25} \mathrm{~N}_{3} \mathrm{O}_{3}$ & 63.93 & 7.89 & 13.16 & 80 & \multirow{2}{*}{-15} \\
\hline & $J[\mathrm{D}]$ & 140 & 319.75 & 63.89 & 7.91 & 13.23 & $\mathrm{~S}_{1}$ & \\
\hline \multirow{2}{*}{$5 e$} & & $120-$ & $\mathrm{C}_{19} \mathrm{H}_{27} \mathrm{~N}_{3} \mathrm{O}_{5}$ & 60.45 & 7.21 & 11.14 & 80 & \multirow{2}{*}{+22.5} \\
\hline & $45[\mathrm{~B}]$ & 122 & 377.95 & 60.42 & 7.20 & 11.21 & $\mathrm{~S}_{2}$ & \\
\hline \multirow{2}{*}{$5 f$} & $54[\mathrm{~B}]$ & $125-$ & $\mathrm{C}_{22} \mathrm{H}_{26} \mathrm{FN}_{3} \mathrm{O}_{3}$ & 66.13 & 6.56 & 10.52 & 74 & \multirow{2}{*}{-17.5} \\
\hline & $62[\mathrm{C}]$ & 130 & 399.47 & 66.23 & 6.59 & 10.59 & $\mathrm{~S}_{2}$ & \\
\hline \multirow{2}{*}{$5 \mathrm{~g}$} & $72[\mathrm{~B}]$ & $183-$ & $\mathrm{C}_{21} \mathrm{H}_{25} \mathrm{~N}_{3} \mathrm{O}_{3}$ & 68.64 & 6.86 & 11.44 & 80 & \multirow{2}{*}{+10} \\
\hline & $75[\mathrm{C}]$ & 184 & 367.44 & 68.90 & 6.75 & 11.52 & $\mathrm{~S}_{2}$ & \\
\hline $5 \mathrm{~h}$ & $63[\mathrm{~B}]$ & $168-$ & $\mathrm{C}_{23} \mathrm{H}_{27} \mathrm{~N}_{3} \mathrm{O}_{5}$ & 64.93 & 6.40 & 9.88 & 55 & -10 \\
\hline $5 \mathrm{n}$ & $68[\mathrm{C}]$ & 170 & 425.48 & 65.11 & 6.26 & 10.97 & $S_{1}$ & -10 \\
\hline & $00[\mathrm{P}]$ & $150-$ & $\mathrm{C}_{26} \mathrm{H}_{26} \mathrm{FN}_{3} \mathrm{O}_{3}$ & 69.78 & 5.86 & 9.39 & 43 & \\
\hline 31 & $90[\mathrm{~B}]$ & 153 & 447.50 & 69.85 & 5.78 & 9.48 & $\mathrm{~S}_{2}$ & +21.5 \\
\hline $5 i$ & $92[\mathrm{~B}]$ & $118-$ & $\mathrm{C}_{23} \mathrm{H}_{27} \mathrm{~N}_{3} \mathrm{O}_{6}$ & 62.57 & 6.16 & 9.52 & 60 & +175 \\
\hline 5j & $92[\mathrm{~B}]$ & 120 & 441.48 & 62.68 & 6.36 & 9.82 & $\mathrm{~S}_{1}$ & $+1 / .5$ \\
\hline & & $125-$ & $\mathrm{C}_{26} \mathrm{H}_{26} \mathrm{FN}_{3} \mathrm{O}_{4}$ & 67.37 & 5.65 & 9.07 & 69 & \\
\hline $5 \mathrm{~K}$ & $66[\mathrm{~B}]$ & 130 & 463.51 & 67.47 & 5.75 & 9.27 & $\mathrm{~S}_{1}$ & -2.5 \\
\hline & & $138-$ & $\mathrm{C}_{16} \mathrm{H}_{22} \mathrm{O}_{4} \mathrm{~N}_{4}$ & 57.47 & 6.63 & 16.76 & 15 & - \\
\hline 51 & $79[\mathrm{~A}]$ & 140 & 334.38 & 57.51 & 6.75 & 16.80 & $S_{1}$ & - \\
\hline & & $168-$ & $\mathrm{C}_{18} \mathrm{H}_{24} \mathrm{~N}_{4} \mathrm{O}_{6}$ & 55.08 & 6.16 & 14.28 & 55 & \\
\hline $5 \mathrm{~m}$ & $30[\mathrm{~B}]$ & 170 & 392.42 & 55.12 & 6.25 & 14.33 & $S_{1}$ & - \\
\hline & & $210-$ & $\mathrm{C}_{21} \mathrm{H}_{23} \mathrm{FN}_{4} \mathrm{O}_{4}$ & 60.86 & 5.59 & 13.52 & 80 & $\ldots$ \\
\hline $5 \mathrm{n}$ & $36[\mathrm{~B}]$ & 215 & 414.43 & 60.88 & 5.66 & 13.55 & $S_{1}$ & - \\
\hline & & $115-$ & $\mathrm{C}_{15} \mathrm{H}_{21} \mathrm{~N}_{3} \mathrm{O}_{3}$ & 61.84 & 7.27 & 14.42 & 65 & \\
\hline 50 & $76[\mathrm{~B}]$ & 120 & 291.35 & 61.89 & 7.23 & 14.37 & $\mathrm{~S}_{1}$ & - \\
\hline $5 n$ & $38[\mathrm{~A}]$ & $149-$ & $\mathrm{C}_{17} \mathrm{H}_{23} \mathrm{~N}_{3} \mathrm{O}_{5}$ & 58.44 & 6.64 & 12.03 & 60 & 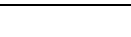 \\
\hline $5 \mathrm{p}$ & $51[\mathrm{C}]$ & 155 & 349.38 & 58.59 & 6.58 & 11.60 & $\mathrm{~S}_{2}$ & - \\
\hline $5 a$ & $65[\mathrm{~B}]$ & $220-$ & $\mathrm{C}_{20} \mathrm{H}_{22} \mathrm{FN}_{3} \mathrm{O}_{3}$ & 64.68 & 5.97 & 11.31 & 85 & 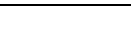 \\
\hline $5 q$ & $65[\mathrm{~B}]$ & 224 & 371.41 & 64.81 & 6.03 & 11.23 & $S_{1}$ & - \\
\hline $5 r$ & $84[\mathrm{~B}]$ & ily & $\mathrm{C}_{18} \mathrm{H}_{27} \mathrm{~N}_{3} \mathrm{O}_{3}$ & 64.84 & 8.16 & 12.60 & 80 & +10 \\
\hline $5 r$ & $84[\mathrm{~B}]$ & only & 333.43 & 64.78 & 8.18 & 12.56 & $\mathrm{~S}_{1}$ & +10 \\
\hline $5 \mathrm{~s}$ & $72[\mathrm{~A}]$ & $140-$ & $\mathrm{C}_{20} \mathrm{H}_{29} \mathrm{~N}_{3} \mathrm{O}_{5}$ & 61.36 & 7.47 & 10.73 & 60 & +20 \\
\hline & & 146 & 391.46 & 61.41 & 7.55 & 10.81 & $\mathrm{~S}_{1}$ & \\
\hline $5 t$ & $86[\mathrm{~B}]$ & 135- & & 66.81 & 6.83 & 10.16 & 55 & -28 \\
\hline & & 140 & 413.49 & 66.85 & 6.86 & 10.14 & $\mathrm{~S}_{1}$ & \\
\hline $5 u$ & $77[\mathrm{~B}]$ & $110-$ & $\mathrm{C}_{17} \mathrm{H}_{24} \mathrm{O}_{4} \mathrm{~N}_{4}$ & 58.61 & 6.94 & 16.08 & 39 & - \\
\hline & $76[\mathrm{C}]$ & 115 & 348.39 & 58.59 & 6.90 & 16.14 & $S_{1}$ & \\
\hline $5 \mathrm{v}$ & $65[\mathrm{~B}]$ & $130-$ & $\mathrm{C}_{19} \mathrm{H}_{26} \mathrm{~N}_{4} \mathrm{O}_{6}$ & 56.15 & 6.45 & 13.79 & 22 & \\
\hline $5 \mathrm{~V}$ & $65[\mathrm{~B}]$ & 135 & 406.43 & 56.21 & 6.37 & 13.86 & $\mathrm{~S}_{1}$ & - \\
\hline & $31[\mathrm{~B}]$ & $198-$ & $\mathrm{C}_{22} \mathrm{H}_{25} \mathrm{FN}_{4} \mathrm{O}_{4}$ & 61.67 & 5.88 & 13.08 & 44 & - \\
\hline $5 \mathrm{w}$ & $55[\mathrm{C}]$ & 200 & 428.46 & 61.65 & 5.92 & 13.15 & $S_{1}$ & - \\
\hline
\end{tabular}


TABLE 2. Spectral data of the prepared compounds (5a-w).

\begin{tabular}{|c|c|c|c|}
\hline $\begin{array}{c}\text { Comp. } \\
\text { No. }\end{array}$ & $\begin{array}{c}{ }^{1} \mathrm{H}-\mathrm{NMR} \\
\left(\delta, \mathrm{ppm}, \mathrm{DMSO}-\mathrm{d}_{6}\right)\end{array}$ & $\begin{array}{c}\text { Mass } \\
{[\mathbf{m} / \mathbf{z},(\%)]}\end{array}$ & $\begin{array}{c}\text { IR } \\
(\mathrm{KBr}) \\
{\left[\mathrm{v}, \mathrm{cm}^{-1}\right]}\end{array}$ \\
\hline $5 \mathrm{a}$ & $\begin{array}{l}2.13\left(\mathrm{~s}, 3 \mathrm{H}, \mathrm{CH}_{3}\right), 2.23-59\left(\mathrm{~m}, 4 \mathrm{H}, 2 \mathrm{CH}_{2}\right), 2.82-3.21(\mathrm{~m}, 4 \mathrm{H}, \\
\left.2 \mathrm{CH}_{2}\right), 4.01\left(\mathrm{~s}, 2 \mathrm{H}, \mathrm{CH}_{2}\right), 6.51(\mathrm{~d}, 1 \mathrm{H}, J=15 \mathrm{~Hz}, \mathrm{CH}=), 7.05(\mathrm{t}, \\
1 \mathrm{H}, J=3.5 \mathrm{~Hz} \text {, furan-4), } 7.33(\mathrm{~d}, 1 \mathrm{H}, J=4.5 \mathrm{~Hz} \text {, furan-3), } 7.5 \\
(\mathrm{d}, 1 \mathrm{H}, J=15 \mathrm{~Hz}, \mathrm{CH}=), 7.6(\mathrm{~d}, 1 \mathrm{H}, J=5 \mathrm{~Hz} \text {, furan-5), } 8.18(\mathrm{~s}, \\
1 \mathrm{H}, \mathrm{NH}, \mathrm{D}_{2} \mathrm{O} \text {-exchangeable). }\end{array}$ & \begin{tabular}{|l|}
$278\left[\mathrm{M}^{+}+1,4\right]$ \\
$277\left[\mathrm{M}^{+}, 24\right]$ \\
$276\left[\mathrm{M}^{+}-1\right.$ \\
$60], 121$ \\
$(100), 93(5)$ \\
\end{tabular} & $\begin{array}{l}3300 \\
(\mathrm{NH}) \\
1699 \\
1660 \\
(\mathrm{CO})\end{array}$ \\
\hline $5 b$ & $\begin{array}{l}1.12\left(\mathrm{t}, J=7.0 \mathrm{~Hz}, 3 \mathrm{H}, \mathrm{CH}_{3}\right), 2.95-3.11\left(\mathrm{~m}, 4 \mathrm{H}, 2 \mathrm{CH}_{2}\right), 3.55- \\
361\left(\mathrm{~m}, 4 \mathrm{H}, 2 \mathrm{CH}_{2}\right), 4.08\left(\mathrm{~s}, 2 \mathrm{H}, \mathrm{CH}_{2}\right), 4.30(\mathrm{q}, J=7.2 \mathrm{~Hz}, 2 \mathrm{H}, \\
\left.\mathrm{CH}_{2}\right), 6.4(\mathrm{~d}, J=15.6 \mathrm{~Hz}, 1 \mathrm{H}, \mathrm{CH}=), 7.35(\mathrm{t}, J=3.5 \mathrm{~Hz}, 1 \mathrm{H}, \\
\text { furan-4), } 7.44(\mathrm{~d}, J=3.6 \mathrm{~Hz}, 1 \mathrm{H}, \text { furan-3), } 7.62(\mathrm{~d}, J=16 \mathrm{~Hz}, \\
1 \mathrm{H}, \mathrm{CH}=), 7.65(\mathrm{~d}, J=5 \mathrm{~Hz}, 1 \mathrm{H}, \text { furan-5), } 8.12(\mathrm{~s}, 1 \mathrm{H}, \mathrm{NH}, \\
\mathrm{D}_{2} \mathrm{O} \text {-exchangeable). }\end{array}$ & \begin{tabular}{l|}
$338\left[\mathrm{M}^{+}+3\right.$ \\
$25]$ \\
$335\left[\mathrm{M}^{+}, 30\right]$ \\
$178(25)$ \\
$150(43)$ \\
$120(100)$
\end{tabular} & $\begin{array}{l}3361(\mathrm{~N} \\
\mathrm{H}) \\
1732 \\
1668 \\
1628 \\
(\mathrm{CO})\end{array}$ \\
\hline $5 c$ & $\begin{array}{l}2.95-3.11\left(\mathrm{~m}, 4 \mathrm{H}, 2 \mathrm{CH}_{2}\right), 3.55-3.61\left(\mathrm{~m}, 4 \mathrm{H}, 2 \mathrm{CH}_{2}\right), 4.11(\mathrm{~s} \\
\left.2 \mathrm{H}, \mathrm{CH}_{2}\right), 6.57-6.59(\mathrm{~m}, 2 \mathrm{H}, \mathrm{Ph}-\mathrm{H}), 6.55(\mathrm{~d}, 1 \mathrm{H}, J=15 \mathrm{~Hz} \\
\mathrm{CH}=), 6.58(\mathrm{t}, 1 \mathrm{H}, J=5.5 \mathrm{~Hz}, \text { furan-4) } 7.05-7.09(\mathrm{~m}, 2 \mathrm{H} \\
\mathrm{Ph}-\mathrm{H}), 7.22(\mathrm{~d}, 1 \mathrm{H}, J=15 \mathrm{~Hz}, \mathrm{CH}=), 7.45(\mathrm{~d}, 1 \mathrm{H}, J=5.5 \mathrm{~Hz} \\
\text { furan-3), 7.58(d, } 1 \mathrm{H}, J=5 \mathrm{~Hz} \text {, furan-5), } 8.31(\mathrm{~s}, 1 \mathrm{H}, \mathrm{NH} \\
\left.\mathrm{D}_{2} \mathrm{O}-\text {-exchangable }\right) .\end{array}$ & $\begin{array}{l}359\left[\mathrm{M}^{+}+2\right. \\
16], 357 \\
\left(\mathrm{M}^{+}, 31\right) \\
252(32) \\
212(71) \\
92(100)\end{array}$ & $\begin{array}{l}3330 \\
(\mathrm{NH}) \\
1698 \\
1614 \\
(\mathrm{CO})\end{array}$ \\
\hline $5 \mathrm{~d}$ & $\begin{array}{l}0.89-0.90\left(\mathrm{~m}, 6 \mathrm{H}, 2 \mathrm{CH}_{3}\right), 2.13\left(\mathrm{~s}, 3 \mathrm{H}, \mathrm{CH}_{3}\right), 2.01-2.15(\mathrm{~m}, \\
1 \mathrm{H}, \mathrm{CH}), 2.32-2.95\left(\mathrm{~m}, 4 \mathrm{H}, 2 \mathrm{CH}_{2}\right), 3.19-3.39(\mathrm{~m}, 4 \mathrm{H}, \\
\left.2 \mathrm{CH}_{2}\right), 4.97(\mathrm{~s}, 1 \mathrm{H}, \mathrm{CH}), 6.36(\mathrm{~d}, 1 \mathrm{H}, J=15 \mathrm{~Hz}, \mathrm{CH}=), 6.48 \\
(\mathrm{t}, 1 \mathrm{H}, J=5.1 \mathrm{~Hz}, \text { furan-4), } 6.83(\mathrm{~d}, 1 \mathrm{H}, J=5 \mathrm{~Hz} \text {, furan-3), } \\
7.35(\mathrm{~d}, 1 \mathrm{H}, J=15 \mathrm{~Hz}, \mathrm{CH}=), 7.78(\mathrm{~d}, 1 \mathrm{H}, J=4.8 \mathrm{~Hz} \text {, furan- } \\
\text { 5), } 8.30\left(\mathrm{~s}, 1 \mathrm{H}, \mathrm{NH}, \mathrm{D}_{2} \mathrm{O} \text {-exchangable). }\right.\end{array}$ & $\begin{array}{l}320\left[\mathrm{M}^{+}+1\right. \\
25], 319 \\
{\left[\mathrm{M}^{+}, 18\right]} \\
318\left[\mathrm{M}^{+}-1\right. \\
9], 121(100) \\
93(25)\end{array}$ & $\begin{array}{l}3320 \\
(\mathrm{NH}) \\
1667 \\
1637 \\
(\mathrm{CO})\end{array}$ \\
\hline $5 \mathrm{e}$ & $\begin{array}{l}0.93\left(\mathrm{~s}, 6 \mathrm{H}, 2 \mathrm{CH}_{3}\right), 1.18\left(\mathrm{t}, 3 \mathrm{H}, J=6.0 \mathrm{~Hz}, \mathrm{CH}_{3}\right), 2.11-2.15 \\
(\mathrm{~m}, 1 \mathrm{H}, \mathrm{CH}), 3.32-3.35\left(\mathrm{~m}, 4 \mathrm{H}, 2 \mathrm{CH}_{2}\right), 3.39-47(\mathrm{~m}, 4 \mathrm{H}, \\
\left.2 \mathrm{CH}_{2}\right), 4.67\left(\mathrm{q}, 2 \mathrm{H}, J=6.9 \mathrm{~Hz}, \mathrm{CH}_{2}\right), 5.5(\mathrm{~s}, 1 \mathrm{H}, \mathrm{CH}), 6.63 \\
(\mathrm{~d}, 1 \mathrm{H}, J=15 \mathrm{~Hz}, \mathrm{CH}=), 6.78(\mathrm{t}, 1 \mathrm{H}, J=5.1 \mathrm{~Hz}, \text { furan-4), } \\
6.83(\mathrm{~d}, 1 \mathrm{H}, J=5 \mathrm{~Hz} \text {, furan-3), } 7.26(\mathrm{~d}, 1 \mathrm{H}, J=15 \mathrm{~Hz}, \mathrm{CH}=), \\
7.78\left(\mathrm{~d}, 1 \mathrm{H}, J=4.8 \mathrm{~Hz} \text {, furan-5), } 8.30\left(\mathrm{~s}, 1 \mathrm{H}, \mathrm{NH}, \mathrm{D}_{2} \mathrm{O}-\right.\right. \\
\text { exchangeable) }\end{array}$ & \begin{tabular}{l|}
$377\left[\mathrm{M}^{+}\right.$ \\
$12]$ \\
$376\left[\mathrm{M}^{+}-1\right.$ \\
$23], 348(4)$ \\
$304(5)$ \\
$276(16)$ \\
$256(100)$
\end{tabular} & $\begin{array}{l}3291 \\
(\mathrm{NH}) \\
1735 \\
1691 \\
1633 \\
(\mathrm{CO})\end{array}$ \\
\hline $5 \mathrm{f}$ & $\begin{array}{c}0.9\left(\mathrm{~s}, 6 \mathrm{H}, 2 \mathrm{CH}_{3}\right), 2.11-2.15(\mathrm{~m}, 1 \mathrm{H}, \mathrm{CH}), 3.51-3.60(\mathrm{~m}, 4 \mathrm{H} \\
\left.2 \mathrm{CH}_{2}\right), 3.71-3.75\left(\mathrm{~m}, 4 \mathrm{H}, 2 \mathrm{CH}_{2}\right), 4.28(\mathrm{~s}, 1 \mathrm{H}, \mathrm{CH}), 6.57- \\
6.59(\mathrm{~m}, 2 \mathrm{H}, \mathrm{Ph}-\mathrm{H}), 6.63(\mathrm{~d}, 1 \mathrm{H}, J=15 \mathrm{~Hz}, \mathrm{CH}=), 6.78(\mathrm{t}, \\
1 \mathrm{H}, J=4.5 \mathrm{~Hz}, \text { furan-4), } 7.05-7.09(\mathrm{~m}, 2 \mathrm{H}, \mathrm{Ph}-\mathrm{H}), 7.22(\mathrm{~d} \\
1 \mathrm{H}, J=15 \mathrm{~Hz}, \mathrm{CH}=), 7.48(\mathrm{~d}, 1 \mathrm{H}, J=4.5 \mathrm{~Hz}, \text { furan-3), } 7.78 \\
\left(\mathrm{d}, 1 \mathrm{H}, J=5 \mathrm{~Hz}, \text { furan-5), } 8.41\left(\mathrm{~s}, 1 \mathrm{H}, \mathrm{NH}, \mathrm{D}_{2} \mathrm{O}-\right.\right. \\
\text { exchangeable }) .\end{array}$ & $\begin{array}{l}400\left[\mathrm{M}^{+}+1,\right. \\
8] \\
399\left(\mathrm{M}^{+}, 55\right] \\
381(100) \\
304(5) \\
219(25)\end{array}$ & $\begin{array}{l}3290( \\
\mathrm{NH}) \\
1657 \\
1623 \\
(\mathrm{CO})\end{array}$ \\
\hline $5 \mathrm{~g}$ & $\begin{array}{l}2.04\left(\mathrm{~s}, 3 \mathrm{H}, \mathrm{CH}_{3}\right), 2.20-2.40\left(\mathrm{~m}, 4 \mathrm{H}, 2 \mathrm{CH}_{2}\right), 2.97\left(\mathrm{~s}, 2 \mathrm{H}, \mathrm{CH}_{2}\right), \\
3.38-3.60\left(\mathrm{~m}, 4 \mathrm{H}, 2 \mathrm{CH}_{2}\right), 5.0-5.05(\mathrm{~m}, 1 \mathrm{H}, \mathrm{CH}), 6.40(\mathrm{~d}, J=16 \\
\mathrm{Hz}, 1 \mathrm{H}, \mathrm{CH}=), 6.57-6.74(\mathrm{~m}, 5 \mathrm{H}, \mathrm{Ph}-\mathrm{H}), 7.26(\mathrm{t}, J=5 \mathrm{~Hz}, 1 \mathrm{H}, \\
\text { furan-4), } 7.38(\mathrm{~d}, J=5.1 \mathrm{~Hz}, 1 \mathrm{H} \text {, furan-3), } 7.40(\mathrm{~d}, J=16 \mathrm{~Hz}, \\
1 \mathrm{H}, \mathrm{CH}=), 7.79(\mathrm{~d}, J=5.1 \mathrm{~Hz}, 1 \mathrm{H}, \text { furan-5), } 8.70(\mathrm{~s}, 1 \mathrm{H}, \mathrm{NH}, \\
\mathrm{D}_{2} \mathrm{O} \text {-exchangeable). }\end{array}$ & $\begin{array}{l}368\left(\mathrm{M}^{+}+1\right. \\
29] \\
367\left[\mathrm{M}^{+}, 68\right] \\
366\left[\mathrm{M}^{+}-1\right. \\
16], 120 \\
(100), 92(46)\end{array}$ & $\begin{array}{l}3140 \\
(\mathrm{NH}) \\
1655 \\
1620 \\
(\mathrm{CO})\end{array}$ \\
\hline $5 \mathrm{~h}$ & $\begin{array}{l}1.18\left(\mathrm{t}, 3 \mathrm{H}, J=6.9 \mathrm{~Hz}, \mathrm{CH}_{3}\right), 2.79-2.90\left(\mathrm{~m}, 4 \mathrm{H}, 2 \mathrm{CH}_{2}\right), 2.97(\mathrm{~s}, \\
\left.2 \mathrm{H}, \mathrm{CH}_{2}\right), 3.75-3.80\left(\mathrm{~m}, 4 \mathrm{H}, 2 \mathrm{CH}_{2}\right), 4.24(\mathrm{q}, 2 \mathrm{H}, J=6.9 \mathrm{~Hz}, \\
\left.\mathrm{CH}_{2}\right), 5.00-5.03(\mathrm{~m}, 1 \mathrm{H}, \mathrm{CH}), 6.50(\mathrm{~d}, J=16 \mathrm{~Hz}, 1 \mathrm{H}, \mathrm{CH}=), \\
6.77-6.89(\mathrm{~m}, 5 \mathrm{H}, \mathrm{Ph}-\mathrm{H}), 7.26(\mathrm{t}, J=5 \mathrm{~Hz}, 1 \mathrm{H}, \text { furan-4), } 7.38(\mathrm{~d}, \\
J=5.1 \mathrm{~Hz}, 1 \mathrm{H}, \text { furan-3), } 7.56(\mathrm{~d}, J=16 \mathrm{~Hz}, 1 \mathrm{H}, \mathrm{CH}=), 7.79(\mathrm{~d}, \\
J=5.1 \mathrm{~Hz}, 1 \mathrm{H}, \text { furan-5), } 8.70\left(\mathrm{~s}, 1 \mathrm{H}, \mathrm{NH}, \mathrm{D}_{2} \mathrm{O}-\text { exchangeable }\right) .\end{array}$ & $\begin{array}{l}396\left(\mathrm{M}^{+}+4,\right. \\
10], 392 \\
{\left[\mathrm{M}^{+}, 20\right]} \\
364(45) \\
277(12) \\
236(100)\end{array}$ & $\begin{array}{l}3278 \\
(\mathrm{NH}) \\
1701 \\
1619 \\
(\mathrm{CO})\end{array}$ \\
\hline
\end{tabular}

Egypt. J. Chem. 56, No.5,6 (2013) 
TABLE 2. (Continued).

\begin{tabular}{|c|c|c|c|}
\hline $\begin{array}{l}\text { Comp. } \\
\text { No. }\end{array}$ & $\begin{array}{c}{ }^{1} \mathrm{H}-\mathrm{NMR} \\
\left(\delta, \mathrm{ppm}, \mathrm{DMSO}-\mathrm{d}_{6}\right)\end{array}$ & $\begin{array}{c}\text { Mass } \\
{[\mathbf{m} / \mathbf{z},(\%)]}\end{array}$ & $\begin{array}{c}\text { IR } \\
(\mathrm{KBr}) \\
{\left[\mathrm{v}, \mathrm{cm}^{-1}\right]}\end{array}$ \\
\hline $5 \mathrm{i}$ & $\begin{array}{l}2.79-2.90\left(\mathrm{~m}, 4 \mathrm{H}, 2 \mathrm{CH}_{2}\right), 3.20\left(\mathrm{~s}, 2 \mathrm{H}, \mathrm{CH}_{2}\right), 3.75-3.80(\mathrm{~m}, 4 \mathrm{H}, \\
\left.2 \mathrm{CH}_{2}\right), 5.2(\mathrm{t}, J=5.7 \mathrm{~Hz}, 1 \mathrm{H}, \mathrm{CH}), 6.50(\mathrm{~d}, J=16 \mathrm{~Hz}, 1 \mathrm{H}, \\
\mathrm{CH}=), 6.70-6.74(\mathrm{~m}, 2 \mathrm{H}, \mathrm{Ph}-\mathrm{H}), 7.07-7.15(\mathrm{~m}, 5 \mathrm{H}, \mathrm{Ph}-\mathrm{H}), 7.13 \\
(\mathrm{~m}, 2 \mathrm{H}, \mathrm{Ph}-\mathrm{H}), 7.24(\mathrm{t}, J=5 \mathrm{~Hz}, 1 \mathrm{H}, \text { furan-4), } 7.38(\mathrm{~d}, J=5.1 \\
\mathrm{Hz}, 1 \mathrm{H}, \text { furan-3), } 7.60(\mathrm{~d}, J=16 \mathrm{~Hz}, 1 \mathrm{H}, \mathrm{CH}=), 7.8(\mathrm{~d}, J=5.1 \\
\mathrm{Hz}, 1 \mathrm{H}, \text { furan-5), } 8.7\left(\mathrm{~s}, 1 \mathrm{H}, \mathrm{NH}, \mathrm{D}_{2} \mathrm{O} \text {-exchangeable). }\right.\end{array}$ & $\begin{array}{l}446\left[\mathrm{M}^{+}, 35\right], \\
387(38), \\
370(11), \\
236(14), \\
201(39), \\
130(100)\end{array}$ & $\begin{array}{l}3277(\mathrm{~N} \\
\mathrm{H}) \\
1664 \\
1655 \\
(\mathrm{CO})\end{array}$ \\
\hline $5 \mathrm{j}$ & $\begin{array}{l}1.18\left(\mathrm{t}, 3 \mathrm{H}, \mathrm{J}=6.9 \mathrm{~Hz}, \mathrm{CH}_{3}\right), 2.79-2.90\left(\mathrm{~m}, 4 \mathrm{H}, 2 \mathrm{CH}_{2}\right), 2.97(\mathrm{~s}, \\
\left.2 \mathrm{H}, \mathrm{CH}_{2}\right) 3.75-3.80\left(\mathrm{~m}, 4 \mathrm{H}, 2 \mathrm{CH}_{2}\right), 4.24(\mathrm{q}, 2 \mathrm{H}, J=6.9 \mathrm{~Hz}, \\
\left.\mathrm{CH}_{2}\right), 5.02-5.06(\mathrm{~m}, 1 \mathrm{H}, \mathrm{CH}), 6.50(\mathrm{~d}, J=16 \mathrm{~Hz}, 1 \mathrm{H}, \mathrm{CH}=), \\
6.77-6.91(\mathrm{~m}, 5 \mathrm{H}, \mathrm{Ph}-\mathrm{H}), 7.26(\mathrm{t}, J=5 \mathrm{~Hz}, 1 \mathrm{H}, \text { furan-4), } 7.38(\mathrm{~d}, \\
J=5.1 \mathrm{~Hz}, 1 \mathrm{H}, \text { furan-3), } 7.56(\mathrm{~d}, J=16 \mathrm{~Hz}, 1 \mathrm{H}, \mathrm{CH}=), 7.79(\mathrm{~d}, \\
J=5.1 \mathrm{~Hz}, 1 \mathrm{H} \text { furan-5), } 8.70\left(\mathrm{~s}, 1 \mathrm{H}, \mathrm{NH}, \mathrm{D}_{2} \mathrm{O}-\text {-exchangeable }\right), \\
9.00\left(\mathrm{~s}, 1 \mathrm{H}, \mathrm{OH}, \mathrm{D}_{2} \mathrm{O} \text { exchangeable). }\right.\end{array}$ & $\begin{array}{l}442\left[\mathrm{M}^{+}+1,3\right] \\
441\left[\mathrm{M}^{+}, 10\right], \\
364(45) \\
278(100) \\
236(25) \\
232(21)\end{array}$ & $\begin{array}{l}3411 \\
(\mathrm{NH}) \\
1702, \\
1632, \\
1611 \\
(\mathrm{CO})\end{array}$ \\
\hline $5 \mathrm{k}$ & 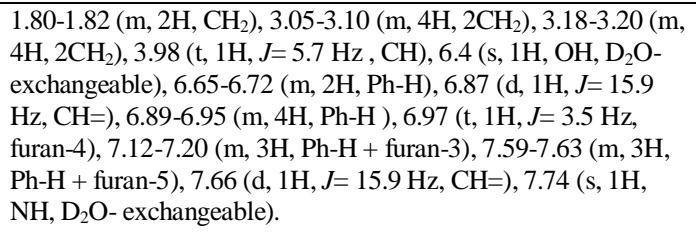 & $\begin{array}{l}442\left[\mathrm{M}^{+}+1\right. \\
3], 441\left[\mathrm{M}^{+}\right. \\
11] \\
364(45) \\
300(100) \\
233(25)\end{array}$ & $\begin{array}{l}3422 \\
(\mathrm{OH}) \\
3130 \\
(\mathrm{NH}), \\
1650 \\
1629 \\
(\mathrm{CO})\end{array}$ \\
\hline 51 & $\begin{array}{l}2.13\left(\mathrm{~s}, 3 \mathrm{H}, \mathrm{CH}_{3}\right), 2.23-2.85\left(\mathrm{~m}, 4 \mathrm{H}, 2 \mathrm{CH}_{2}\right), 3.55-3.60(\mathrm{~m}, 4 \mathrm{H}, \\
\left.2 \mathrm{CH}_{2}\right), 4.15\left(\mathrm{~s}, 2 \mathrm{H}, \mathrm{CH}_{2}\right), 4.32\left(\mathrm{~s}, 2 \mathrm{H}, \mathrm{CH}_{2}\right), 6.21(\mathrm{~d}, 1 \mathrm{H}, J=15 \\
\mathrm{Hz}, \mathrm{CH}=), 6.56(\mathrm{t}, 1 \mathrm{H}, J=5.5 \mathrm{~Hz}, \text { furan-4), } 6.70(\mathrm{~d}, 1 \mathrm{H}, J=2.5 \\
\mathrm{Hz} \text {, furan-3), } 7.12(\mathrm{~d}, 1 \mathrm{H}, J=16 \mathrm{~Hz}, \mathrm{CH}=), 7.15(\mathrm{~d}, 1 \mathrm{H}, J=5 \\
\mathrm{Hz} \text {, furan-5), } 8.12\left(\mathrm{~s}, 2 \mathrm{H}, 2 \mathrm{NH}, \mathrm{D}_{2} \mathrm{O} \text {-exchangeable }\right) .\end{array}$ & $\begin{array}{l}335\left[\mathrm{M}^{+}+1\right. \\
9], 334\left[\mathrm{M}^{+}\right. \\
12], 333\left[\mathrm{M}^{+}-\right. \\
1,14], 277 \\
(100), 137(73)\end{array}$ & $\begin{array}{l}3326, \\
3149 \\
(\mathrm{NH}), \\
1660, \\
1661 \\
(\mathrm{CO}) .\end{array}$ \\
\hline $5 \mathrm{~m}$ & $\begin{array}{l}1.20\left(\mathrm{t}, 3 \mathrm{H}, J=7.0 \mathrm{~Hz}, \mathrm{CH}_{3}\right), 3.55-3.60\left(\mathrm{~m}, 4 \mathrm{H}, 2 \mathrm{CH}_{2}\right), 3.85- \\
3.90\left(\mathrm{~m}, 4 \mathrm{H}, \mathrm{CH}_{2}\right), 4.1\left(\mathrm{q}, 2 \mathrm{H}, J=7.0 \mathrm{~Hz}, \mathrm{CH}_{2}\right) 4.20(\mathrm{~s}, 2 \mathrm{H}, \\
\left.\mathrm{CH}_{2}\right), 4.30\left(\mathrm{~s}, 2 \mathrm{H}, \mathrm{CH}_{2}\right), 6.40(\mathrm{~d}, 1 \mathrm{H}, J=15 \mathrm{~Hz}, \mathrm{CH}=), 6.56(\mathrm{t}, \\
1 \mathrm{H}, J=5.0 \mathrm{~Hz} \text {, furan-4), } 6.70(\mathrm{~d}, 1 \mathrm{H}, J=2.5 \mathrm{~Hz}, \text { furan-3), } 7.15 \\
(\mathrm{d}, 1 \mathrm{H}, J=5 \mathrm{~Hz} \text {, furan-5), } 7.52(\mathrm{~d}, 1 \mathrm{H}, J=16 \mathrm{~Hz}, \mathrm{CH}=), 8.32(\mathrm{~s}, \\
2 \mathrm{H}, 2 \mathrm{NH}, \mathrm{D}_{2} \mathrm{O} \text {-exchangeable). }\end{array}$ & $\begin{array}{l}393\left[\mathrm{M}^{+}+1\right. \\
3], 392\left(\mathrm{M}^{+}\right. \\
17), 348(21) \\
236(23) \\
121(100)\end{array}$ & $\begin{array}{l}3348, \\
3149 \\
(\mathrm{NH}) \\
1689 \\
1653 \\
1626 \\
(\mathrm{CO}) \\
\end{array}$ \\
\hline $5 n$ & $\begin{array}{l}\text { 3.06- } 3.12\left(\mathrm{~m}, 4 \mathrm{H}, 2 \mathrm{CH}_{2}\right), 3.60-3.65\left(\mathrm{~m}, 4 \mathrm{H}, 2 \mathrm{CH}_{2}\right), 4.13 \\
\left(\mathrm{~s}, 2 \mathrm{H}, \mathrm{CH}_{2}\right), 4.15\left(\mathrm{~s}, 2 \mathrm{H}, \mathrm{CH}_{2}\right), 6.58(\mathrm{~d}, 1 \mathrm{H}, J=16 \mathrm{~Hz}, \\
\mathrm{CH}=), 6.80-6.89(\mathrm{~m}, 2 \mathrm{H}, \mathrm{Ph}-\mathrm{H}), 7.00(\mathrm{~d}, 1 \mathrm{H}, J=3.5 \mathrm{~Hz}, \\
\text { furan-4), } 7.05-7.08(\mathrm{~m}, 3 \mathrm{H}, \mathrm{Ph}-\mathrm{H}+\text { furan-3), } 7.26(\mathrm{~d}, 1 \mathrm{H}, J= \\
15.9 \mathrm{~Hz}, \mathrm{CH}=), 7.79(\mathrm{~d}, 1 \mathrm{H}, J=4.5 \mathrm{~Hz} \text {, furan-5), } 8.36(\mathrm{~s}, \\
2 \mathrm{H}, 2 \mathrm{NH}, \mathrm{D}_{2} \mathrm{O} \text { exchangeable). }\end{array}$ & $\begin{array}{l}415\left[\mathrm{M}^{+}+1\right. \\
1], 414\left[\mathrm{M}^{+}\right. \\
13], 357(28) \\
219(52) \\
120(100)\end{array}$ & $\begin{array}{l}3278 \\
2099 \\
(\mathrm{NH}) \\
1656 \\
1635 \\
(\mathrm{CO})\end{array}$ \\
\hline 50 & $\begin{array}{l}2.01\left(\mathrm{~s}, 1 \mathrm{H}, \mathrm{CH}_{3}\right), 2.30\left(\mathrm{~s}, 3 \mathrm{H}, \mathrm{CH}_{3}\right), 3.06-3.11(\mathrm{~m}, 4 \mathrm{H}, \\
\left.2 \mathrm{CH}_{2}\right), 3.20-3.25\left(\mathrm{~m}, 4 \mathrm{H}, 2 \mathrm{CH}_{2}\right), 4.12(\mathrm{t}, J=7.1 \mathrm{~Hz}, 2 \mathrm{H}, \\
\left.\mathrm{CH}_{2}\right), 6.08(\mathrm{t}, J=4.5 \mathrm{~Hz}, 1 \mathrm{H}, \text { furan-4), } 6.25(\mathrm{~d}, J=15 \mathrm{~Hz}, \\
1 \mathrm{H}, \mathrm{CH}=), 6.86(\mathrm{~d}, J=4.6 \mathrm{~Hz}, 1 \mathrm{H}, \text { furan-3), } 7.10(\mathrm{~d}, J=16 \\
\mathrm{Hz}, 1 \mathrm{H}, \mathrm{CH}=), 8.15\left(\mathrm{~s}, 1 \mathrm{H}, \mathrm{NH}, \mathrm{D}_{2} \mathrm{O} \text { - exchangeable }\right) .\end{array}$ & $\begin{array}{l}293\left[\mathrm{M}^{+}+1\right. \\
35), 292\left[\mathrm{M}^{+}\right. \\
6], 277 \\
(25) 191(18) \\
133(100)\end{array}$ & $\begin{array}{l}3250 \\
(\mathrm{NH}), \\
1659 \\
1627 \\
(\mathrm{CO})\end{array}$ \\
\hline $5 p$ & $\begin{array}{l}1.12\left(\mathrm{t}, J=6.0 \mathrm{~Hz}, 3 \mathrm{H}, \mathrm{CH}_{3}\right), 2.14\left(\mathrm{~s}, 3 \mathrm{H}, \mathrm{CH}_{3}\right), 2.21-3.11 \\
\left(\mathrm{~m}, 4 \mathrm{H}, 2 \mathrm{CH}_{2}\right), 3.49-3.51\left(\mathrm{~m}, 4 \mathrm{H}, 2 \mathrm{CH}_{2}\right), 4.02(\mathrm{t}, J=7.1 \mathrm{~Hz}, \\
\left.2 \mathrm{H}, \mathrm{CH}_{2}\right), 4.30\left(\mathrm{q}, J=6.2 \mathrm{~Hz}, 2 \mathrm{H}, \mathrm{CH}_{2}\right), 6.15(\mathrm{t}, J=3.5 \mathrm{~Hz}, \\
1 \mathrm{H}, \text { furan-4), } 6.39(\mathrm{~d}, J=15.6 \mathrm{~Hz}, 1 \mathrm{H}, \mathrm{CH}=), 6.58(\mathrm{~d}, J=3.6 \\
\mathrm{Hz}, 1 \mathrm{H}, \text { furan-3), } 7.10(\mathrm{~d}, J=16 \mathrm{~Hz}, 1 \mathrm{H}, \mathrm{CH}=), 8.01(\mathrm{~s}, 1 \mathrm{H}, \\
\mathrm{NH}, \mathrm{D}_{2} \mathrm{O}-\text { exchangeable). }\end{array}$ & $\begin{array}{l}350\left[\mathrm{M}^{+}, 15\right], \\
349\left[\mathrm{M}^{+}-1,\right. \\
6), 276(100), \\
192(19), \\
135(23) .\end{array}$ & $\begin{array}{l}3350 \\
(\mathrm{NH}), \\
1695, \\
1664 \\
(\mathrm{CO}) .\end{array}$ \\
\hline
\end{tabular}


TABLE 2. (Continued).

\begin{tabular}{|c|c|c|c|}
\hline $\begin{array}{l}\text { Comp. } \\
\text { No. }\end{array}$ & $\begin{array}{c}{ }^{1} \mathrm{H}-\mathrm{NMR} \\
\left(\delta, \mathrm{ppm}, \mathrm{DMSO}-\mathrm{d}_{6}\right)\end{array}$ & $\begin{array}{c}\text { Mass } \\
{[\mathbf{m} / \mathbf{z},(\%)]}\end{array}$ & $\begin{array}{c}\text { IR } \\
(\mathrm{KBr}) \\
{\left[\mathrm{v}, \mathrm{cm}^{-1}\right]}\end{array}$ \\
\hline $5 q$ & $\begin{array}{l}2.30\left(\mathrm{~s}, 3 \mathrm{H}, \mathrm{CH}_{3}\right), 2.98-3.05\left(\mathrm{~m}, 4 \mathrm{H}, 2 \mathrm{CH}_{2}\right), 3.55-3.70(\mathrm{~m}, \\
\left.4 \mathrm{H}, 2 \mathrm{CH}_{2}\right), 4.14\left(\mathrm{~s}, 2 \mathrm{H}, \mathrm{CH}_{2}\right), 6.23-6.33(\mathrm{~m}, 2 \mathrm{H}, \mathrm{Ph}-\mathrm{H}), 6.48 \\
(\mathrm{~d}, 1 \mathrm{H}, J=16 \mathrm{~Hz}, \mathrm{CH}=), 6.65(\mathrm{t}, 1 \mathrm{H}, J=4 \mathrm{~Hz} \text { furan-4), 6.70- } \\
6.75(\mathrm{~m}, 2 \mathrm{H}, \mathrm{Ph}-\mathrm{H}), 6.98(\mathrm{~d}, 1 \mathrm{H}, J=5 \mathrm{~Hz}, \text { furan-3), } 7.09(\mathrm{~d}, \\
1 \mathrm{H}, J=5 \mathrm{~Hz} \text {, furan-5), } 7.12 \mathrm{~d}, 1 \mathrm{H}, J=15 \mathrm{~Hz}, \mathrm{CH}=), 8.20(\mathrm{~s}, \\
, 1 \mathrm{H}, \mathrm{NH}, \mathrm{D}_{2} \mathrm{O} \text { exchangeable). }\end{array}$ & $\begin{array}{l}372\left[\mathrm{M}^{+}+1\right. \\
16], 371 \\
{\left[\mathrm{M}^{+}, 46\right]} \\
276(9) \\
192(11) \\
135(100)\end{array}$ & $\begin{array}{l}3330 \\
(\mathrm{NH}), \\
1698 \\
1651 \\
(\mathrm{CO})\end{array}$ \\
\hline $5 \mathrm{r}$ & $\begin{array}{l}0.97-1.0\left(\mathrm{~m}, 6 \mathrm{H},, 2 \mathrm{CH}_{3}\right), 2.12-2.15(\mathrm{~m}, 1 \mathrm{H}, \mathrm{CH}), 2.14(\mathrm{~s}, 3 \mathrm{H}, \\
\left.\mathrm{CH}_{3}\right), 3.32-3.35\left(\mathrm{~m}, 4 \mathrm{H}, 2 \mathrm{CH}_{2}\right), 3.39\left(\mathrm{~m}, 4 \mathrm{H}, 2 \mathrm{CH}_{2}\right), 4.24(\mathrm{~s}, \\
1 \mathrm{H}, \mathrm{CH}), 6.31(\mathrm{~d}, 1 \mathrm{H}, J=15 \mathrm{~Hz}, \mathrm{CH}=), 6.14(\mathrm{t}, 1 \mathrm{H}, J=5.1 \mathrm{~Hz} \text {, } \\
\text { furan-4), } 6.33(\mathrm{~d}, 1 \mathrm{H}, J=5 \mathrm{~Hz} \text {, furan-3), } 7.16(\mathrm{~d}, 1 \mathrm{H}, J=15 \\
\mathrm{Hz}, \mathrm{CH}=), 8.11\left(\mathrm{~s}, 1 \mathrm{H}, \mathrm{NH}, \mathrm{D}_{2} \mathrm{O} \text { exchangeable }\right) .\end{array}$ & $\begin{array}{l}320\left[\mathrm{M}^{+}+1\right. \\
10], 319 \\
{\left[\mathrm{M}^{+}, 35\right]} \\
304(100) \\
221(75)\end{array}$ & $\begin{array}{l}3316 \\
(\mathrm{NH}), \\
1655 \\
1632 \\
(\mathrm{CO})\end{array}$ \\
\hline $5 \mathrm{~s}$ & $\begin{array}{l}0.86-0.89\left(\mathrm{~m}, 6 \mathrm{H}, 2 \mathrm{CH}_{3}\right), 1.18\left(\mathrm{t}, 3 \mathrm{H}, J=6.0 \mathrm{~Hz}, \mathrm{CH}_{3}\right), 2.0- \\
2.01(\mathrm{~m}, 1 \mathrm{H}, \mathrm{CH}), 2.38\left(\mathrm{~s}, 3 \mathrm{H}, \mathrm{CH}_{3}\right), 3.32-3.35(\mathrm{~m}, 4 \mathrm{H}, \\
\left.2 \mathrm{CH}_{2}\right), 3.39-3.44\left(\mathrm{~m}, 4 \mathrm{H}, 2 \mathrm{CH}_{2}\right), 4.05(\mathrm{q}, 2 \mathrm{H}, J=6.9 \mathrm{~Hz}, \\
\left.\mathrm{CH}_{2}\right), 4.64(\mathrm{~s}, 1 \mathrm{H}, \mathrm{CH}), 6.51(\mathrm{~d}, 1 \mathrm{H}, J=15 \mathrm{~Hz}, \mathrm{CH}=), 6.64(\mathrm{t}, \\
1 \mathrm{H}, J=5.1 \mathrm{~Hz}, \text { furan-4), } 6.83(\mathrm{~d}, 1 \mathrm{H}, J=5.0 \mathrm{~Hz}, \text { furan-3), } 7.16 \\
(\mathrm{d}, 1 \mathrm{H}, J=15 \mathrm{~Hz}, \mathrm{CH}=), 8.32\left(\mathrm{~s}, 1 \mathrm{H}, \mathrm{NH}, \mathrm{D}_{2} \mathrm{O} \text { exchangeable). }\right.\end{array}$ & $\begin{array}{l}392\left[\mathrm{M}^{+}+1\right. \\
4], 391 \\
{\left[\mathrm{M}^{+}, 12\right]} \\
362(54) \\
134(100)\end{array}$ & $\begin{array}{l}3323 \\
(\mathrm{NH}), \\
1701, \\
1663, \\
1642 \\
(\mathrm{CO})\end{array}$ \\
\hline $5 \mathrm{t}$ & $\begin{array}{l}\text { 0.98-1.0 (m, 6H, 2CH3), 2.05-2.15 (m, 1H, CH), } 2.42(\mathrm{~s}, 3 \mathrm{H}, \\
\mathrm{CH} 3), 3.32-3.35(\mathrm{~m}, 4 \mathrm{H}, 2 \mathrm{CH} 2), 3.38-3.41(\mathrm{~m}, 4 \mathrm{H}, 2 \mathrm{CH} 2), \\
4.54(\mathrm{~s}, 1 \mathrm{H}, \mathrm{CH}), 6.22(\mathrm{~m}, 2 \mathrm{H}, \mathrm{Ph}-\mathrm{H}), 6.51(\mathrm{~d}, 1 \mathrm{H}, \mathrm{J}=3.5 \mathrm{~Hz}, \\
\text { furan-4), 6.57 (d, 1H, J= 15.8 Hz, CH=), 6.87-7.04 (m, 3H, } \\
\mathrm{Ph}-\mathrm{H}), 7.12(\mathrm{~d}, 1 \mathrm{H}, \mathrm{J}=5 \mathrm{~Hz}, \text { furan-3), } 7.17(\mathrm{~d}, 1 \mathrm{H}, \mathrm{J}=15.9 \mathrm{~Hz}, \\
\mathrm{CH}=), 8.18(\mathrm{~s}, 2 \mathrm{H}, 2 \mathrm{NH}, \mathrm{D} 2 \mathrm{O} \text { exchangeable }) .\end{array}$ & $\begin{array}{l}415[\mathrm{M}++1, \\
10], 414[\mathrm{M}+, \\
14], 413 \\
{[\mathrm{M}+-1,35]} \\
207(18) \\
136(100)\end{array}$ & $\begin{array}{l}3369 \\
(\mathrm{NH}), \\
1642 \\
1610 \\
(\mathrm{CO})\end{array}$ \\
\hline $5 \mathrm{u}$ & $\begin{array}{l}1.91(\mathrm{~s}, 1 \mathrm{H}, \mathrm{CH} 3), 2.31(\mathrm{~s}, 3 \mathrm{H}, \mathrm{CH} 3), 3.36-3.41(\mathrm{~m}, 4 \mathrm{H}, \\
2 \mathrm{CH} 2), 3.63(\mathrm{~m}, 4 \mathrm{H}, 2 \mathrm{CH} 2), 4.09(\mathrm{~s}, 2 \mathrm{H}, \mathrm{CH} 2), 4.27(\mathrm{~s}, 2 \mathrm{H}, \\
\mathrm{CH} 2), 6.21(\mathrm{t}, \mathrm{J}=3.7 \mathrm{~Hz}, 1 \mathrm{H}, \text { furan-4), } 6.59(\mathrm{~d}, \mathrm{~J}=15.6 \mathrm{~Hz}, \\
1 \mathrm{H}, \mathrm{CH}=), 6.65(\mathrm{~d}, \mathrm{~J}=4.6 \mathrm{~Hz}, 1 \mathrm{H}, \text { furan-3), } 7.18(\mathrm{~d}, \mathrm{~J}=15.0 \\
\mathrm{Hz}, 1 \mathrm{H}, \mathrm{CH}=), 8.19(\mathrm{~s}, 2 \mathrm{H}, 2 \mathrm{NH}, \mathrm{D} 2 \mathrm{O}-\text { exchageable). }\end{array}$ & $\begin{array}{l}349[\mathrm{M}++1, \\
2], 348 \\
{[\mathrm{M}+, 10)} \\
286(46) \\
190(23) \\
135(100) \\
\end{array}$ & $\begin{array}{l}3438 \\
3119 \\
(\mathrm{NH}) \\
1658 \\
1617 \\
(\mathrm{CO})\end{array}$ \\
\hline $5 v$ & $\begin{array}{l}1.17(\mathrm{t}, 3 \mathrm{H}, \mathrm{J}=7.0 \mathrm{~Hz}, \mathrm{CH} 3), 2.30(\mathrm{~s}, 1 \mathrm{H}, \mathrm{CH} 3), 3.56-3.60(\mathrm{~m}, \\
4 \mathrm{H}, 2 \mathrm{CH} 2), 3.65-3.70(\mathrm{~m}, 4 \mathrm{H}, 2 \mathrm{CH} 2), 4.07(\mathrm{q}, 2 \mathrm{H}, \mathrm{J}=7.0 \mathrm{~Hz}, \\
\mathrm{CH} 2), 4.20(\mathrm{~s}, 2 \mathrm{H}, \mathrm{CH}), 6.21(\mathrm{t}, 1 \mathrm{H}, \mathrm{J}=5.0 \mathrm{~Hz} \text {, furan-4), } 6.46 \\
(\mathrm{d}, 1 \mathrm{H}, \mathrm{J}=15 \mathrm{~Hz}, \mathrm{CH}=), 6.66(\mathrm{~d}, 1 \mathrm{H}, \mathrm{J}=2.5 \mathrm{~Hz} \text {, furan-3), } 7.15 \\
(\mathrm{d}, 1 \mathrm{H}, \mathrm{J}=16 \mathrm{~Hz}, \mathrm{CH}=), 8.24(\mathrm{~s}, 2 \mathrm{H}, 2 \mathrm{NH}, \mathrm{D} 2 \mathrm{O}- \\
\text { exchangeable). }\end{array}$ & $\begin{array}{l}407[\mathrm{M}+, 10] \\
378(9) \\
349(100) \\
191(21) \\
134(62)\end{array}$ & $\begin{array}{l}3350, \\
3120 \\
(\mathrm{NH}) \\
1696 \\
1663 \\
(\mathrm{CO}) \\
\end{array}$ \\
\hline $5 w$ & $\begin{array}{l}2.32(\mathrm{~s}, 1 \mathrm{H}, \mathrm{CH} 3), 3.05-3.16(\mathrm{~m}, 4 \mathrm{H}, 2 \mathrm{CH} 2), 3.35-3.59(\mathrm{~m}, \\
4 \mathrm{H}, 2 \mathrm{CH} 2), 4.11(\mathrm{~s}, 2 \mathrm{H}, \mathrm{CH} 2), 4.13(\mathrm{~s}, 2 \mathrm{H}, \mathrm{CH} 2), 6.22(\mathrm{~m}, \\
2 \mathrm{H}, \mathrm{Ph}-\mathrm{H}), 6.50(\mathrm{~d}, 1 \mathrm{H}, \mathrm{J}=3.5 \mathrm{~Hz} \text {, furan-4), } 6.66(\mathrm{~d}, 1 \mathrm{H}, \mathrm{J}= \\
13.8 \mathrm{~Hz}, \mathrm{CH}=), 6.99-7.04(\mathrm{~m}, 2 \mathrm{H}, \mathrm{Ph}-\mathrm{H}), 7.10(\mathrm{~d}, 1 \mathrm{H}, \mathrm{J}=5 \\
\mathrm{Hz}, \text { furan-3), } 7.18(\mathrm{~d}, 1 \mathrm{H}, \mathrm{J}=13.9 \mathrm{~Hz}, \mathrm{CH}=), 8.26(\mathrm{~s}, 2 \mathrm{H}, \\
\text { 2NH, D2O-exchangeable). }\end{array}$ & $\begin{array}{l}429[\mathrm{M}+, 5], \\
317(100), \\
285(3), \\
191(29), \\
135(37) .\end{array}$ & $\begin{array}{l}3293 \\
3075 \\
(\mathrm{NH}) \\
1655 \\
1623 \\
(\mathrm{CO})\end{array}$ \\
\hline
\end{tabular}

\section{Biological evaluation}

All the obtained products 5a-w have been investigated for their anti-allergic and anti-inflammatory activities. All compounds showed anti-allergic effects, the highest and greatest capacities are recorded for compounds $5 \mathrm{~g}, 5 \mathrm{~h}, 5 \mathrm{j}, 5 \mathrm{k}, 5 \mathrm{~m}, 5 \mathrm{n}, 5 \mathrm{v}$ and $5 \mathrm{w}$ relative to the anti-allergic activity of Loratadine ${ }^{\circledR}$ (Clarinix) (Table 3). 
The evaluation of anti-inflammatory activity was carried out according to the method of Winter et al. ${ }^{(16,17)}$, where the inhibitory activity of the studied compounds on carrageen an-induced rat's paw edema. Most of these compounds have no anti-inflammatory activities except $5 \mathrm{~d}, 5 \mathrm{e}$ and $5 \mathrm{p}$ which showed the relative activities of $65.12,75.12$ and $69.18 \%$, respectively. This means that derivatives comprised valinyl-residues have promised anti-inflammatory activities. Also, the presence of methyl group in $\mathrm{N}$-3-(5-methyl)-2-furanyl acrylic acid has no valuable effect on the biological activity.

TABLE 3. Anti-allergic capacities and anti-inflammatory activities of compounds (5a-w).

\begin{tabular}{|c|c|c|c|}
\hline Compd. No. & $\begin{array}{l}\% \text { Inhibition in } \\
\text { contraction }\end{array}$ & $\begin{array}{l}\text { \% Protection against } \\
\text { edema (at } 2 \mathrm{mg} / \mathrm{Kg} \text { ) }\end{array}$ & $\underset{\mathrm{mg} / \mathrm{Kg}}{\mathrm{LD}_{50}}$ \\
\hline $5 \mathrm{a}$ & $71 \pm 0.66$ & NA & $3145.76 \pm 4.56$ \\
\hline $5 b$ & $75 \pm 0.32$ & NA & $1236.87 \pm 4.55$ \\
\hline $5 \mathrm{c}$ & $78 \pm 0.64$ & NA & $2113.67 \pm 2.39$ \\
\hline $5 \mathrm{~d}$ & $76 \pm 0.32$ & $65.12 \pm 0.43$ & $3456.67 \pm 3.45$ \\
\hline $5 \mathrm{e}$ & $70 \pm 0.56$ & $57.12 \pm 0.44$ & $2167.66 \pm 2.11$ \\
\hline $5 f$ & $76 \pm 0.77$ & NA & $3009 \pm 4.58$ \\
\hline $5 \mathrm{~g}$ & $86 \pm 0.32$ & NA & $2567.8 \pm 2.44$ \\
\hline $5 \mathrm{~h}$ & $86 \pm 0.32$ & NA & $2642.21 \pm 2.31$ \\
\hline $5 \mathrm{i}$ & $88 \pm 0.65$ & NA & $28765.67 \pm 4.16$ \\
\hline $5 \mathrm{j}$ & $99 \pm 0.09$ & NA & $2376 \pm 2.44$ \\
\hline $5 \mathrm{k}$ & $99 \pm 0.32$ & NA & $2468 \pm 5.44$ \\
\hline 51 & $71 \pm 0.66$ & NA & $3145.76 \pm 4.56$ \\
\hline $5 \mathrm{~m}$ & $88 \pm 0.32$ & NA & $2556.38 \pm 5.66$ \\
\hline $5 n$ & $87 \pm 0.36$ & NA & $2222.34 \pm 2.28$ \\
\hline 50 & $73 \pm 0.64$ & NA & $3214.67 \pm 3.56$ \\
\hline $5 p$ & $75 \pm 0.43$ & $69.18 \pm 0.43$ & $509.21 \pm 5.59$ \\
\hline $5 q$ & $77 \pm 0.32$ & $40.55 \pm 0.32$ & $456.76 \pm 4.59$ \\
\hline $5 \mathrm{r}$ & $72 \pm 0.32$ & NA & $987.21 \pm 2.57$ \\
\hline $5 \mathrm{~s}$ & $74 \pm 0.31$ & NA & $3425.87 \pm 5.39$ \\
\hline $5 \mathrm{t}$ & $79 \pm 0.34$ & $42.121 \pm 0.23$ & $2134.67 \pm 4.68$ \\
\hline $5 \mathrm{u}$ & $80 \pm 0.89$ & NA & $2698.65 \pm 2.55$ \\
\hline $5 v$ & $85 \pm 0.56$ & NA & $2589 \pm 2.56$ \\
\hline $5 w$ & $81 \pm 0.43$ & NA & $2564.4 \pm 4.34$ \\
\hline Loratidine $^{(\circledR)}$ & $71 \pm 0.04$ & --- & $2250.25 \pm 2.26$ \\
\hline Diclofenac $^{\circledR}$ & --- & 70.14 & $2160.25 \pm 1.35$ \\
\hline
\end{tabular}

NA= No activity 


\section{Experimental}

Melting points were determined in open glass capillaries using an Electrothermal IA 9000 SERIES digital melting point apparatus (Electrothermal, UK) and are uncorrected. Optical rotations were measured by a "Polax-D" polarimeter (ATAGO), provided by a SI-Na-1 Sodium lamp. Microanalyses were performed for all final compounds on an Elementar-Vario EL (Elementar-Vario EL, Germany) (Micro-analytical Unit, Central Services Laboratory, National Research Centre, Cairo, Egypt). The ${ }^{1} \mathrm{H}-\mathrm{NMR}$ spectra were recorded on a Varian Mercury VX-300 NMR spectrometer (Varian, USA). ${ }^{1} \mathrm{H}-\mathrm{NMR}$ spectra were run at $300 \mathrm{MHz}$ in DMSO- $d_{6}$ as solvent. Chemical shifts $\delta$ are quoted in ppm and were related to that of the solvents. Mass spectra were recorded on a Shimadzu GCMS-QP 1000EX (EI, 70 eV) (Shimadzu, Japan) and Hewlett-Packard (EI, 70 eV) (Hewlett-Packard, USA). IR spectra were obtained with a Brucker-Vector 22 (Bruker Rhein-Stetten, Germany). All the reactions were monitored using thin layer chromatography (TLC) using silica gel aluminum sheets $60 \mathrm{~F}_{254}$ (Merck). While "S" stands for a chromatographic solvent system of chloroform/ methanol/ acetic acid, 85/10/5 by volume, $S_{1}$ and $S_{2}$ represent the same solvent system to which petroleum ether $\left(40-60^{\circ} \mathrm{C}\right)$ was added, in an equal or half ratio volume, respectively.

Synthesis of N-[(4-substituted-1-piperazinyl)-oxo-(alkyl and ethycarbamoyl)]-3(2-furanyl) acrylamides (5a-n) and 3-(5-methyl-2-furanyl) acrylamides (5o-w) (General procedures)

[i] Acid Chloride method [Procedure A]

Triethylamine $(2.42 \mathrm{~g} \equiv 3.4 \mathrm{ml}, 24 \mathrm{mmol})$ was added in portions to a well stirred cold solution $\left(0-5^{\circ} \mathrm{C}\right)$ of acid derivatives $4 \mathrm{a}$ and $4 \mathrm{~h}(12 \mathrm{mmol})$ and with the appropriate substituted piperazine $(12 \mathrm{mmol})$ in THF/DCM $(1: 4,150 \mathrm{ml})$. The reaction mixture was stirred for $3 \mathrm{hr}$ at the same temperature with simultaneous adjustment of $\mathrm{pH} \approx 8$. The TEA hydrochloride was filtered off, excess solvent distilled off and residue in, mainly, ethyl acetate (EtOAc) was washed with $\mathrm{HCl}$ $(1 \mathrm{~N}, 3 \times 20 \mathrm{ml}), \mathrm{NaHCO}_{3}(5 \%, 3 \times 20 \mathrm{ml}), \mathrm{H}_{2} \mathrm{O}(3 \times 20 \mathrm{ml})$ and dried over anhydrous sodium sulfate. Excess solvent was distilled off to afford $5 \mathrm{a}, \mathrm{b}, 5 \mathrm{l}, 5 \mathrm{p}$ and $5 \mathrm{~s}$, in good yields (Table 1).

[ii] In situ active ester method [Procedure B]

A solution of DCC ( $3 \mathrm{~g}, 15 \mathrm{mmol})$ in THF $(10 \mathrm{ml})$ was added, over $30 \mathrm{~min}$, to a stirred cold solution $\left(0-5^{\circ} \mathrm{C}\right)$ of the acid product $4 \mathrm{~b}-\mathrm{g}(15 \mathrm{mmol})$ and $\mathrm{N}$ hydroxybenzotriazole (HOBt) $(1.7 \mathrm{~g}, 15 \mathrm{mmol})$ in THF $(50 \mathrm{ml})$. Stirring was continued for $15 \mathrm{~min}$ at the same temperature, and a mixture of the appropriate substituted piperazine $(15 \mathrm{mmol})$ and TEA $(1.5 \mathrm{~g} \equiv 2.13 \mathrm{ml}, 15 \mathrm{mmol})$ in THF $(50 \mathrm{ml})$ was then added. Stirring was continued at the same temperature for $3 \mathrm{hr}$, at $\mathrm{pH} \approx 8\left(\mathrm{Et}_{3} \mathrm{~N}\right)$ and at $0^{\circ} \mathrm{C}$ overnight, then at room temperature for $24 \mathrm{hr}$. Drops of glacial acetic acid were added to the cold $\left(0^{\circ} \mathrm{C}\right)$ suspension and the reaction mixture was filtered off. The filtrate was evaporated and the residue was taken in

acetonitrile $(10 \mathrm{ml})$ and then kept in cold and the formed urea (DCHU) was Egypt. J. Chem. 56, No.5,6 (2013) 
separated by filtration. Solvent was distilled off under reduced pressure and the residue was taken up in EtOAc, washed with $\mathrm{NaHCO}_{3}(1 \mathrm{~N}, 3 \times 10 \mathrm{ml})$, distilled water $(3 \times 10 \mathrm{ml}), \mathrm{KHSO}_{4}(5 \%, 3 \times 10 \mathrm{ml})$, distilled water $(3 \times 10 \mathrm{ml})$ and dried over anhydrous sodium sulfate. The dried and decolorized EtOAc solution was concentrated $(5 \mathrm{ml})$ and chromatographically purified on manually prepared preparative TLC plates to get the products 5a-k, 5m-r and 5t-w (Table 1).

[iii] Mixed anhydride method [Procedure C]

Ethyl chloroformate $(1.08 \mathrm{~g} \equiv 0.95 \mathrm{ml}, 10 \mathrm{mmol})$ was added to a stirred and cold $\left(-15^{\circ} \mathrm{C}\right)$ THF solution of the acid product $4 \mathrm{~g}$ or $4 \mathrm{~h}(10 \mathrm{mmol})$ and $\mathrm{N}$ methylmorpholine $(\sim 1 \mathrm{ml}, 10 \mathrm{mmol})$. The reaction mixture was stirred for additional $10 \mathrm{~min}$ and then cold solution of appropriate substituted piperazine (10 $\mathrm{mmol})$ in THF $(30 \mathrm{ml})$ was added. Stirring was maintained for $3 \mathrm{hr}$ at $\left(-15^{\circ} \mathrm{C}\right)$ then for $12 \mathrm{hr}$ at room temperature. The solvent was evaporated and the residue was taken into EtOAc $(30 \mathrm{ml})$ then washed with $\mathrm{KHSO}_{4}(3 \%, 3 \times 10 \mathrm{ml})$, distilled water $(3 \times 10 \mathrm{ml}), \mathrm{NaHCO}_{3}(3 \%, 3 \times 10 \mathrm{ml})$ and finally with water $(3 \times 10 \mathrm{ml})$ then dried over anhydrous sodium sulfate. The solvent was evaporated to dryness and the obtained residue triturated with $\mathrm{n}$-hexane. The obtained solid was collected by filtration and crystallized from ethanol/n-hexane to afford $5 \mathrm{a}, 5 \mathrm{c}, 5 \mathrm{f}, 5 \mathrm{~g}, 5 \mathrm{~h}$, $5 \mathrm{p}, 5 \mathrm{u}$ and $5 \mathrm{w}$ as identified by melting point, mixed melting point and TLC in comparison with samples prepared according to procedure B (Table 1).

\section{Biological activities}

The animal design model involved in this study is measuring the antianaphylactic activities due to histamine release.

\section{Anti-allergic activity}

\section{Evaluation}

The percent inhibition of spasmogen induced contraction is calculated. The percentage of inhibition in contraction (due to histamine release) is the anti-allergic potency, which is compared to that of Loratadine ${ }^{\circledR}$ (standard reference drug).

\section{Procedure}

Albino guinea pigs of either sex weighing 300-450 g are sacrificed with an overdose of ether. The chest cavity is opened and the lungs are removed. They were cut into strips of $5 \mathrm{~cm}$ and placed into a physiological saline solution. Thereafter, the lung strips are mounted in an organ bath containing a nutritive solution. The bath was bubbled with carbogen and maintained at $37^{\circ} \mathrm{C}$ under a pre-load of $0.5 \mathrm{~g}-3 \mathrm{~g}$; the tissue was left to equilibrate for $30-60 \mathrm{~min}$. Prior to testing carbachol is added to the bath to test the lung strips ability of contraction. Twenty minutes later, two values are obtained by adding the spasmogen.

- Histamine dihydrochloride $10^{-6} \mathrm{~g} / \mathrm{ml}$ for $5 \mathrm{~min}$,

- Ca-ionophore $5 \times 10^{-6} \mathrm{~g} / \mathrm{ml}$ for $5 \mathrm{~min}$, or

- Leukotriene $\mathrm{LTC}_{4}\left(10^{-9}-10^{-8}\right) \mathrm{g} / \mathrm{ml}$ for $10 \mathrm{~min}$,

To the bath and recording the contractile force at its maximal level. Following 
$20 \mathrm{~min}$, equilibration period resulted, the spasmogen is administrated again, $5 \mathrm{~min}$ there after and the test compound is added in cumulative dose from $10^{-8}-10^{-4} \mathrm{~g} / \mathrm{ml}$ at 5 or $10 \mathrm{~min}$ intervals. The contractile response is determined isometrically ${ }^{(15)}$ (Table 3).

\section{Evaluation of anti-inflammatory activity}

The inhibitory activity of the studied compounds on carrageenin-induced rat's paw edema was carried out according to the method of Winter et al. ${ }^{(16,17)}$.

Groups of adult male albino rats (150-180 gm), each of 8 animals were orally dosed with the test compounds at a dose level of 2.5 and $5 \mathrm{mg} / \mathrm{kg}$ an hour before carrageenin challenge.

Foot paw edema was induced by sub planter injection of $0.05 \mathrm{ml}$ of $1 \%$ suspension of carrageenin in saline into the planter tissue of one hind paw. An equal volume of saline was injected to the other hind paw and served as control. Four hours after drug administration the animal was decapitated, blood was collected and the paws were rapidly excised.

The average weight of edema was estimated for the treated as well as the control group and the percentage inhibition of weight of edema was also evaluated then percentage protection against edema was estimated (Table 3). Diclophenac $^{\circledR} \quad(2.5$ and $5 \mathrm{mg} / \mathrm{kg})$ was employed as standard reference against which the test compounds were compared.

Estimation of plasma prostaglandin $E_{2}\left(P G E_{2}\right)$

Heparinized blood samples were collected from rats $(n=8)$, plasma was separated by centrifugation at $12000 \mathrm{~g}$ for $2 \mathrm{~min}$ at $4^{\circ} \mathrm{C}$ and immediately stored frozen $20^{\circ} \mathrm{C}$ until use.

The designs correlate-EIA prostaglandin in $\mathrm{E}_{2}\left(\mathrm{PGE}_{2}\right)$ kit is a competitive immune assay for the quantitative determination of $\mathrm{PGE}_{2}$ in biological fluids. The kit uses a monoclonal antibody to $\mathrm{PGE}_{2}$ to bind, in a competitive manner, the $\mathrm{PGE}_{2}$ in the sample. After a simultaneous incubation at room temperature the excess reagents were washed away and the substrate was added. After a short incubation time the enzyme reaction was stopped and the yellow color generated was read on a micro plate reader (DYNATCH, MR 5000) at $405 \mathrm{~nm}$. The intensity of the bound yellow color is inversely proportional to the concentration of $\mathrm{PGE}_{2}$ in either standard or samples. The percentage inhibition of plasma $\mathrm{PGE}_{2}$ for each compound was estimated (Table 3).

\section{Evaluation of acute toxicity study}

The test compounds were administered orally at different dose levels in separate groups of animals. After $24 \mathrm{hr}$ of drug administration percent mortality in each group was observed. From the data obtained, the lethal dose $\left(\operatorname{LD}_{50}\right)$ was calculated by the method of Austen et al. ${ }^{(18)}$ (Table 3).

Egypt. J. Chem. 56, No.5,6 (2013) 


\section{References}

1. Nishikawa, Y., Shindo, T., Ishii, K., Nakamoara, H., Kon, T. and Uno, H., Acryamide derivatives as antiallergic agents. I. Synthesis and structure-activity relationships of $N$-[(4-substituted 1-piperazinyl)Alkyl]-3-(Aryl and heteroaryl) acrylamides. Chem. Pharm. Bull. 37, 100-105 (1989).

2. Nishikawa, Y., Shindo, T., Ishii, K., Nakamoura, H., Kon, T. and Uno, H., Acrylamide derivatives as antiallergic agents. 2. Synthesis and structure-activity relationships of $\mathrm{N}$-[4-[4-(diphenylmethyl) piperazinyl]-butyl]-3-(3-Pyridyl)Acrylamides. J. Med. Chem. 32, 583-593 (1989).

3. Nishikawa, Y., Shindo, T., Ishii, K., Nakamoura, H., Kon, T. and Uno, H., Acrylamide derivatives as antiallergic agents. iii. synthesis and structure-activity relationships of $\mathrm{N}$ - [4-(4-(diphenylmethyl-1-piperazinyl)-butyl]- and $\mathrm{N}$-[4-(4(diphenylmethylene-1-piperidyl)-Butyl]-3-heteroacrylamides. Chem. Pharm. Bull. 37, 684-687 (1989).

4. Mager, P.P., QSAR, diagnostic statistics, and molecular modelling of antiallergic acrylamide derivatives. Drug Des. Discov. 9(2), 107-18 (1992).

5. Ishii, K., Yakuo, I., Motoyoshi, S., Nakagawa, H. and Nakamure, H., Inhibition of leukotriene production by $\mathrm{N}$-[4-[4-(diphenylmethyl)-1-piperazinyl]butyl]-3-(6methyl-3-pyridyl)acrylamide (AL-3264, a new antiallergic agent. Jpn. J. Pharmacol. 65, 19-25 (1995).

6. Yakuo, I., Ishii, K., Sakaki, C., Nakamure, H. and Takeyama, K. , Inhibitory effect of AL-3264, a new antiallergic agent, on the prausnitz-Kustner reaction and Leukotriene production in monkeys. Jpn. J. Pharmacol. 67, 263-266 (1995).

7. Ishii, K., Yokuo, I. and Nakamura, H., Dissociation between central and peripheral antihistamine activities of the new antiallergic agent $N$-4-[4-(4-diphenylmethyl)-1piperazinyl]-butyl]-3-(6-methyl-3-pyridyl)acrylamide in rats and monkeys. Arzneim. Forsch. 43(6), 668-671 (1993).

8. Hassal, C.H., Johnson, W. H. and Theobald, C. J., Amino-acids and peptides, Part 21. Synthesis of a congener of the cyclohexadepsipeptide antibiotic, monamycin. $J$. Chem. Soc. Perkin Trans, 1, 1451-1459 (1979).

9. Alewood, P.F., Perich, J.W.I. and Johns, R.B., Synthesis of a protected phosphoamino acid, N $\alpha$-tert- butyloxycarbonyl- $O$ - diethylphosphoro -L-serine. Synth. Commun. 12, 821-828 (1982).

10. Shalaby, A.M. and El-Eraky, W., Studies on $O$-[2,6-dichlorophenyl-1-amino]phenyl acetic acid I. Syn- thesis, antiinflammatory, analgesic and ulcerogenic activities of some new amino acid conjugates. Il Farmaco, 52 (2), 83-87(1997).

11. Abo-Ghalia, M.H. and Soliman, A.M., Synthesis and antischistosomal activity of a new 2-nicotinoyl dipeptide analogue of praziquantel. Arzneim. Forsch, Drug Research, 46 (1), 207-209 (1996).

12. Shalaby, A.M. El-Sawy, A.A. , Abd-El Salam, O.I., Abdullah, M. and Elshihaby, Egypt. J. Chem. 56, No.5,6 (2013) 
S.A., Amino acid and peptide derivatives as anti-allergic and anti-inflammatory agents. Egypt. J. Chem. 51(6), 807-821 (2008).

13. Abo-Glalia, M.H., Shalaby, A.M., El-Eraky, W. and Awad, H.M., Synthesis and anti-phlogistic potency of some new non-proteiogenic amino acid conjugates of 'diclofenac,. Amino Acids, 16, 425 (1999).

14. Abd El-Salam, O.I., Shalaby, A.M., El-Sawy, A.A., Elshihaby, S.A. and Abdulla, M., Synthesis of some $N$-[(4-substituted-1-piperazinyl)-oxo(alkyl and ethylcarbamoyl)]-3-(2-thiophenyl)acrylamides as non-steroidal anti-allergic and antiinflammatory agents. Open J. Synth. Theo. Appl. (OJSTA), 2, 63-72 (2013).

15. Ferman, J.C., Shelly, R. and Webber, S.E., Contraction of guinea-pig lung parenchymal strips by substance $\mathrm{p}$ and related peptides. Arch. Int. Pharmacol. 278, 193-206 (1985).

16. Winter, C.A. , Risely, E.A. and Nuss, G.W., Carrageenin- induced edema in hind paw of the rat as an assay for antiiflammatory drugs. Proc. Soc. Exp. Biol. Med. 111, 541 (1962).

17. Winter, C.A., Risely, E.A. and Nuss, G.W., Anti-in- flammatory and antipyretic activities of indomethacin, 1-(p-chlorobenzoyl)-5-methoxy-2-methylindole-3-Acetic acid. J. Pharmacol. Exp. Ther. 141, 369-376 (1963).

18. Austen, K.F. and Brocklehurst, W. E., Anaphylaxis in chopped guiena pig lung: i. efect of peptidase substrates and inhibitors. J. Exp. Med. 113, 521-539 (1961).

(Received 17/11/2013

accepted 29/12/ 2013) 


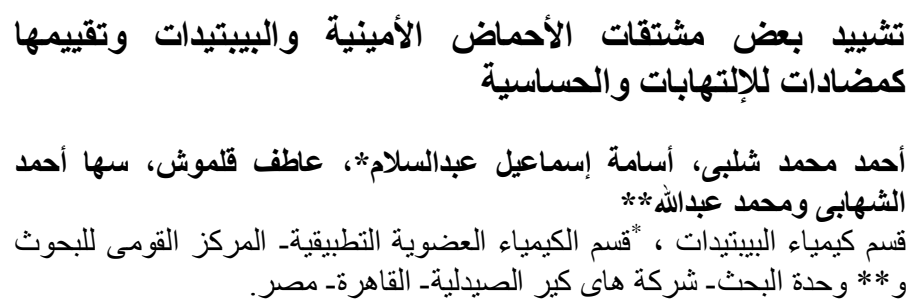

تم تشييد سلسلة جديدة من الأميدات 5a-w بتفاعل ن-3-3-(2)-فيور انيل)أكريلويل،

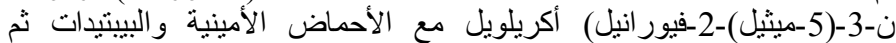

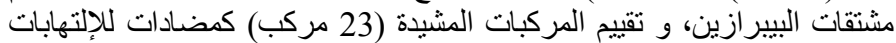
و الحساسية مقارنة بكل من الفولتارين و اللور اتيدين.

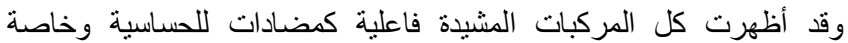

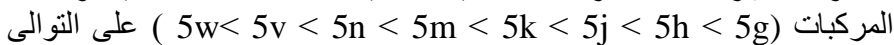

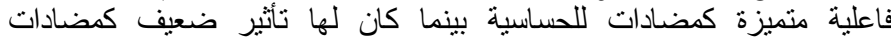
للإلتهابات و أثنتت تحاليل السمية أنها أمنة وغير سامة. 